\title{
Um olhar crítico sobre o estudo do desamparo aprendido 1
}

\author{
Maria Helena Leite Hunziker ${ }^{2}$ \\ Departamento de Psicologia Experimental Universidade de São Paulo
}

O desamparo aprendido vem sendo observado numa grande variedade de espécies. Esse artigo mostra as características básicas dos experimentos sobre desamparo aprendido realizados com sujeitos infra-humanos. A análise crítica da maioria desses experimentos revela problemas de procedimento e mensuração do fenômeno, além de pouca precisão nas definições empregadas. É descrito um procedimento modificado, o qual produziu resultados mais precisos quanto à aprendizagem operante em estudo, se comparados com os resultados dos procedimentos convencionais. Sugere-se que a maior precisão dos experimentos sobre desamparo aprendido trará importantes contribuições para a análise do comportamento em geral, e para a investigação do controle aversivo e dos efeitos da não-contingência, em particular.

Palavras chave: desamparo aprendido, comportamento animal, controle aversivo, estímulos incontroláveis

\begin{abstract}
A critical view about the learned helplessness studies

Learned helplessness effect has been observed across a wide range of species. This paper introduces the basic characteristics of learned helplessness experiments performed with infrahuman subjects. A critical analysis of most of these experiments shows procedural and measurement problems in addition to a low defitional precision. Some results produced by a modified procedure are reported, which were able to generate more accurate data (compared to conventional procedures) with respect to the operant learning under study. It is proposed that well established learned helplessness experiments can make significant contributions to behavior analysis, particularly to the investigation of aversive control and to the effects of non-contingency.
\end{abstract}

Key words: learned helplessness, animal behavior, aversive control, uncontrollable stimuli

Ao se falar sobre o desamparo aprendido é necessário que se faça a distinção entre o fenômeno comportamental e uma das suas hipóteses explicativas. O desamparo aprendido, enquanto efeito comportamental (learned helplessness effect), caracteriza-se pela dificuldade de aprendizagem demonstrada por sujeitos previamente expostos a eventos aversivos incontroláveis. ${ }^{3}$ Essa dificuldade é avaliada em comparação ao comportamento apresentado por sujeitos ingê-

1. O conteúdo deste texto baseia-se na conferência apresentada no IV Latini Dies, congresso realizado em Guadalajara (México), em 23-26 de fevereiro de 1995.

2. A autora é pesquisadora do CNPq (processo no. 523612/95-8).

3. Conforme se verá mais adiante, essa caracterização do desamparo pode sofrer algumas variações. Contudo, a "dificuldade de aprendizagem" é a mais freqüentemente utilizada.

Endereço para correspondência:Departamento de Psicologia Experimental Instituto de Psicologia - USP Av. Prof. Mello Moraes, 1721, CEP 05508-900, São Paulo, SP E-mail: hunziker@usp.br 
nuos (não submetidos previamente a nenhum tratamento específico), submetidos a igual condição de aprendizagem. Analisam-se diferentes aspectos do comportamento, tais como a latência de fuga/esquiva ou a quantidade de exposições à contingência necessária para se obter o padrão de aprendizagem.

Enquanto explicação do comportamento, a hipótese do desamparo aprendido (learned helplessness hypothesis) diz que esse efeito é determinado pela aprendizagem de que há uma independência entre respostas e estímulos, aprendizagem essa que se estabelece frente aos eventos incontroláveis e se generaliza para novas situações (Maier e Seligman, 1976; Seligman, Maier e Solomon, 1971).

A denominação comum entre comportamento e hipótese produz não apenas imprecisões de análise, como gera também um viés teórico. Em primeiro lugar, ela obscurece o fato de que tal hipótese é apenas uma dentre várias propostas para explicar o comportamento. Além disso, ela pode dar a falsa ilusão de que o fenômeno já está explicado, desfavorecendo a busca de maiores evidências para sua confirmação, ou mesmo de outras explicações alternativas. Portanto, para garantir maior precisão de análise, neste texto o termo desamparo aprendido será sempre utilizado em referência ao comportamento, sendo explicitado quando ele se referir à hipótese teórica.

O estudo do desamparo aprendido teve início dentro do referencial teórico e metodológico da análise funcional do comportamento, onde se investigam as relações entre eventos do organismo (R) e do ambiente (S). As contingências operantes (de dois termos) são definidas pela probabilidade com que estímulos (S) ocorrem após a emissão de uma classe de respostas específica (R), ou seja, p(S/R). Embora nem sempre explicitada, a contingência especifica também a probabilidade de $\mathrm{S}$ ocorrer na ausência dessa classe de respostas (NR), ou seja, $\mathrm{p}(\mathrm{S} / \mathrm{NR})$. Por exemplo, no esquema de reforçamento contínuo feito em laboratório, o reforço é sempre apresentado após a emissão de uma determinada classe de respostas (tal como pressão à barra), mas nunca na sua ausência: esse é um arranjo experimental onde $\mathrm{p}(\mathrm{S} / \mathrm{R})=1,0$ e $\mathrm{p}(\mathrm{S} / \mathrm{NR})=0,0$. Nesse caso ambas as probabilidades são diferentes entre si, sendo que esse arranjo pode ser traduzido na expressão $\mathrm{p}(\mathrm{S} / \mathrm{R}) \mathrm{p}(\mathrm{S} / \mathrm{NR})$.

A diferença entre ambas as probabilidades define uma contingência operante, sendo variável a magnitude dessa diferença (no exemplo citado tivemos a magnitude máxima uma vez que foram utilizados os valores extremos de variação de probabilidade). Nesses casos, dizse que a ocorrência da resposta controla a ocorrência do estímulo que a segue, o que esse estímulo é contingente à resposta. Assim, estímulos contingentes são também chamados de estímulos controláveis. Inversamente, nas relações onde ambas as probabilidades são iguais entre si, o estímulo ocorre independentemente da ocorrência (ou ausência) da resposta, o que lhe confere a denominação de estímulo não-contingente ou estímulo incontrolável.

A sensibilidade dos indivíduos às conseqüências do seu comportamento vem sendo apontada como um dos principais mecanismos de adaptação das diferentes espécies (Skinner, 1981). Se o controle do ambiente permite a construção de um repertório comportamental adaptativo, qual a conseqüência para o indiví- 
duo do seu contato com eventos ambientais incontroláveis? Essa é a questão subjacente aos estudos sobre o desamparo aprendido. ${ }^{4}$

Os estudos pioneiros consistiram na exposição de cães a choques elétricos incontroláveis, seguida (24hs após) de um treino operante de fuga/esquiva. Observou-se que esses sujeitos não aprenderam a resposta de fuga/esquiva, ao contrário dos animais não expostos previamente aos choques (ou expostos aos mesmos choques, porém controláveis), que a aprenderam rapidamente. Como apenas a experiência prévia com choques não bastava para explicar esses resultados, o efeito de interferência na aprendizagem foi atribuído à incontrolabilidade dos choques (Overmier e Seligman, 1967; Seligman e Maier, 1967).

Esse efeito foi replicado com diversas espécies, dentre mamíferos, aves, peixes e insetos, o que lhe confere grande generalidade (Eisenstein e Carlson, 1997). Contudo, deve-se destacar que esse efeito vem sendo investigado no laboratório animal quase que exclusivamente com eventos aversivos incontroláveis (mais especificamente, choques elétricos). Essa predominância provavelmente se explique pelo fato do estudo do desamparo ter surgido dentro de uma linha de investigação sobre esquiva, quando alunos de Richard Solomon - um dos defensores da "teoria de dois fatores" para a esquiva (Rescorla e Solomon, 1967) - testavam essa "teoria" (ver Peterson, Maier e Seligman.,

4. Não se pretende, com isso, dizer que o estudo do desamparo deu início à investigação dos efeitos de eventos aversivos não contingentes. A supressão condicionada, por exemplo, foi estudada por Estes e Skinner (1941) varias décadas antes do estudo do desamparo aprendido. O objetivo aqui é apenas destacar ao leitor a questão básica subjacente ao estudo do desamparo.
1993, que mostram o início "acidental" do estudo do desamparo). Histórico à parte, um fato $\mathrm{a}$ ser analisado criticamente é que a generalização do desamparo para situações não aversivas deve ser cuidadosa. Apesar de alguns autores argumentarem que ela pode ser feita (Peterson et al., 1993), deve-se considerar que tais generalizações baseiam-se em poucos (e freqüentemente questionáveis) dados experimentais, insuficientes para sua sustentação. Numa análise mais rigorosa, pode-se dizer que ainda hoje é questionável se eventos incontroláveis não aversivos produzem interferência em aprendizagens futuras, ou mesmo se eventos aversivos incontroláveis interferem em novas aprendizagens sob reforçamento positivo. Esse é um aspecto que necessita de mais investigação.

Outro problema associado ao estudo do desamparo está na inconsistência da sua caracterização: ora ele é apontado como "não aprendizagem de novas respostas", ora como "não aprendizagem de fuga", e às vezes como "dificuldade de aprendizagem" (Maier e Seligman, 1976). Conforme analisado por Levis (1976), essas diferentes denominações representam processos comportamentais distintos. Segundo esse autor, os termos "não aprendizagem" e "não aprendizagem de fuga" sugerem diferentes graus de generalidade para o fenômeno: insensibilidade a qualquer contingência ou apenas a contingências de reforçamento negativo, respectivamente. Além disso, o efeito caracterizado como "não aprendizagem" sugere uma incapacidade de aprender, diferentemente de "dificuldade de aprendizagem" que é um efeito menos drástico. Ou seja, dependendo da caracterização do fenômeno, ele se torna mais ou menos generalizável, ou representa diferentes 
graus de dificuldade na adaptação do indivíduo a novos contextos ambientais.

A ambigüidade conceitual é indesejável na ciência. A falta de precisão na caracterização desse fenômeno tem permitido que sejam igualmente classificados como desamparo aprendido comportamentos submetidos a diferentes tipos de controle. Conseqüentemente, uma análise crítica do conjunto de publicações na área revela, muitas vezes, uma aparente falta de coerência dos resultados obtidos. Uma conceituação mais rigorosa do fenômeno seria, portanto, um primeiro requisito indispensável para se estabelecer a coerência interna na área.

Levando-se em conta que os estudos vem sendo realizados quase que exclusivamente com eventos aversivos incontroláveis, e que a aprendizagem é avaliada sob reforçamento negativo, pode-se sugerir que uma definição mais cuidadosa do fenômeno explicite esses aspectos. Por exemplo, o desamparo seria mais adequadamente definido como "dificuldade de aprendizagem sob reforçamento negativo em função da experiência prévia com eventos aversivos incontroláveis". Embora mais restritiva, essa definição se ajusta aos dados experimentais existentes até o momento, impedindo generalizações aparentemente estimulantes, porém sem base experimental que as sustente.

$\mathrm{O}$ baixo rigor conceitual e metodológico nessa área de investigação reflete-se, também, na aplicação clínica que foi dada ao desamparo aprendido. Pouco tempo após os primeiros estudos, o desamparo aprendido foi sugerido como um modelo animal de depressão (Seligman, 1975). Essa transposição dos dados básicos do laboratório animal para a clínica se deu sem que o fenômeno estivesse solidamente estabelecido. Como conseqüência, a grande quantidade de trabalhos que relaciona o desamparo a essa psicopatologia são experimentalmente questionáveis. Por exemplo, na psicofarmacologia, o desamparo aprendido vem sendo utilizado como simples teste de drogas antidepressivas, sem destaque para a análise das variáveis ambientais que controlam os comportamentos investigados. Além disso, nessa literatura existe uma grande diversidade de procedimentos que vem sendo utilizados como "fórmulas" de produção de comportamentos rotulados como desamparo, os quais não são equivalentes entre si se analisados funcionalmente (comparar, por exemplo, os procedimentos empregados por Hemingway e Reigle, 1987; Kametani, Kimura e Shimizu, 1983; Martin, Gozlan e Puech, 1992; Petty e Sherman, 1979).

Outra inconsistência que pode ser observada principalmente nos estudos voltados à aplicação clínica, relaciona-se ao uso indevido do termo desamparo. Originalmente de cunho meramente descritivo de um desempenho que ocorre sob determinadas condições, ele algumas vezes é utilizado (equivocadamente) com sentido causal: diz-se que o indivíduo se comporta de tal maneira porque está desamparado, e não que ele está desamparado porque se comporta de tal maneira. Tanto essa imprecisão, como a grande diversidade de comportamentos (controlados por diferentes condições) que passaram a ser rotulados indiscriminadamente de desamparo aprendido, talvez decorram da falta de análise do comportamentos em questão, e tem como conseqüência o fato de que essa denominação tenha se tornado inespecífica no uso clínico (Peterson et al., 1993).

Esses problemas conceituais tem afastado do estudo do desamparo os pesquisadores interessados no desenvolvimento da análise 
funcional do comportamento. Isso apenas agravou a falta de rigor metodológico, criando um círculo vicioso. Contudo, a importância teórica das questões subjacente ao estudo do desamparo justifica que os analistas do comportamento retomem a sua investigação e proponham soluções para esses problemas.

Do ponto de vista metodológico, o aspecto mais frágil desses estudos tem sido a caracterização da variável dependente: apesar do desamparo ser definido como dificuldade de aprendizagem, as contingências utilizadas nos estudos com animais (ratos, em sua maioria) freqüentemente não permitem a análise desse processo. Após o tratamento experimental (choques incontroláveis ou nenhum choque), o teste de aprendizagem de fuga vem sendo realizado utilizando-se a contingência de reforçamento negativo em FR2 para a resposta de correr na shuttlebox (Maier et al., 1973), ou FR3 para a resposta de pressão à barra (Seligman, Rosellini e Kozak., 1975). Com ambas as contingências, observa-se em vários estudos que os animais ingênuos (não submetidos a qualquer tratamento prévio) apresentaram latências inalteradas ou aumentadas ao longo da sessão (ver Alloy e Bersh, 1979, grupos N; Freda e Klein, 1976, grupo R , experimento 1; Jackson, Maier e Coon, 1979, grupo restrained, experimento 1A; Maier et al., 1973, grupos restrained, experimentos 1A, 1B e 3; Maier e Testa,1975, grupos NPS-BOTH e NPS-Follows). Ora, se a aprendizagem de fuga é caracterizada pela redução da latência da resposta que produz o término do evento aversivo, não se pode dizer que esses animais tenham aprendido. Por não haverem sido submetidos a qualquer manipulação experimental, esses animais são os que deveriam fornecer o referencial de aprendizagem so- bre o qual se avaliaria a interferência da incontrolabilidade: a ausência de aprendizagem mostrada por esses sujeitos impede, portanto, que essa análise seja feita (ver em Hunziker, 1981, análise mais detalhada sobre esse problema).

Por mais que esse fato seja conflitante com a própria definição do fenômeno, é surpreendente a constatação de que ele tem passado "desapercebido". No geral, os autores tem utilizado como medida do desamparo apenas a maior latência apresentada na sessão de fuga pelos sujeitos expostos aos choques incontroláveis, desprezando o fato de que se não houve aprendizagem não se pode analisar a interferência dos choques incontroláveis sobre ela. Rigorosamente, a única conclusão possível de se tirar desses estudos é que os choques incontroláveis tornaram os indivíduos mais lentos na emissão da resposta que estava sendo avaliada. Contudo, essa lentidão pode refletir apenas um efeito motor decorrente das alterações neuroquímicas induzidas pelos choques incontroláveis (conforme proposto por Weiss, Glazer e Pohorecky, 1976). Embora alterações motoras e neuroquímicas sejam inegavelmente relevantes como efeito da incontrolabilidade dos choques, suas implicações teóricas diferem daquelas postuladas pela hipótese do desamparo aprendido, cuja ênfase recai sobre a menor sensibilidade às contingências operantes. Por mais óbvio que pareça, é necessário que seja lembrado que para se estudar os efeitos da incontrolabilidade sobre processos de aprendizagem é indispensável que essa aprendizagem possa ser claramente mensurada.

Esse foi o ponto de partida de uma série de estudos desenvolvidos no nosso laboratório. Nos primeiros experimentos, testamos os procedimentos descritos nos principais trabalhos 
sobre o desamparo aprendido. Numa primeira fase, um grupo de ratos $(n=8)$ foi exposto a 60 choques elétricos incontroláveis de $1,0 \mathrm{~mA}$ e $10,0 \mathrm{~s}$ de duração fixa, ministrados a intervalos médios de $1 \mathrm{~min}$ (grupo experimental); um segundo grupo de animais não recebeu qualquer choque (grupo ingênuo). Após 24 hs, todos os sujeitos foram igualmente submetidos à sessão de teste de aprendizagem de fuga, com 60 choques (de igual intensidade e distribuição temporal dos anteriores) cuja interrupção era contingente à emissão de diferentes respostas, para os diferentes grupos de sujeitos $(n=8)$ : para a resposta de correr, foram utilizadas contingências de reforçamento em FR1 e FR2; para a resposta de pressionar a barra, utilizou-se a contingência de reforçamento em FR3. As latências de fuga desses animais foram comparadas às apresentadas por sujeitos ingênuos submetidos a iguais contingências. Os resultados revelaram que os animais tratados com choques incontroláveis foram os que apresentaram as maiores latências de fuga. Apesar desse resultado aparentemente confirmado do desamparo, notou-se que: 1) o uso da contingência de fuga FR1 (para a resposta de correr na shuttlebox) produziu latências muito baixas desde o início da sessão de teste, o que impossibilitou a avaliação do controle operante do comportamento, que seria caracterizado pelo decréscimo das mesmas ao longo da sessão; 2) as contingências de FR2 (para a resposta de correr de um compartimento ao outro da shuttlebox) e FR3 (para a resposta de pressão à barra) também não produziram um padrão comportamental de aprendizagem, nos sujeitos ingênuos, embora as suas latências iniciais tenham sido relativamente elevadas, elas se mantiveram praticamente inalteradas ao longo da sessão. Assim, apesar dos animais expos- tos previamente aos choques incontroláveis terem apresentado latências mais altas que seus pares ingênuos, não se pode concluir pela replicação do desamparo aprendido como fenômeno de interferência num processo de aprendizagem (Hunziker, 1977).

Nossa análise dos resultados com a reposta de correr partiram do fato de que durante um choque essa resposta tem uma alta probabilidade de ser eliciada dado que é uma resposta espécie-específica (Bolles, 1970). Assim, independentemente do controle operante, as latências iniciais já são bastante baixas. Para se observar o controle operante de uma resposta numa contingência de fuga é necessário que ela seja emitida com altas latências iniciais, de forma que a sua redução gradual (em função do término do choque ser contingente a ela) evidencie o reforçamento negativo. Portanto, algo precisaria ser modificado no procedimento utilizado com os testes na shuttlebox. Fizemos uma pequena alteração na própria caixa experimental, introduzindo uma divisória da shuttlebox com um pequeno orifício a $8,0 \mathrm{~cm}$ acima do piso, em substituição à divisória convencional que tem esse orifício ao nível do piso. Com essa pequena alteração, para que o animal passasse ao compartimento oposto da shutlebox, era preciso saltar através do orifício, e não mais simplesmente correr para a extremidade oposta da caixa Como a resposta de saltar ocorre inicialmente com altas latências, foi possível registrar claramente a sua redução na medida em que o sujeito ingênuo se expunha à contingência de fuga. Com isso, pudemos ter um parâmetro comparativo dessa aprendizagem operante que nos permitiu avaliar a dificuldade de aprendizagem apresentada pelos animais previamente expostos a choques incontroláveis. Com esse procedi- 
mento foi replicado o fenômeno básico de desamparo aprendido com ratos, sem os problemas apontados anteriormente (Hunziker, 1981).

O desamparo observado com esse nosso arranjo experimental vem sendo replicado por diferentes pesquisadores (Graeff, 1991; Damiani, 1993; Gouveia Jr, 1996; Mestre, 1996; Yano, 1996). Porém, apesar de replicar o fenômeno básico, esse procedimento gerou alguns resultados divergentes dos descritos na literatura para algumas manipulações efetuadas. Por exemplo, diversos autores apontaram a dificuldade de se obter desamparo em ratos utilizandose reforçamento negativo contínuo (FR1). Segundo eles, o desamparo apenas seria obtido com ratos em situações de aprendizagem de respostas "difíceis", sendo esse "difícil" caracterizado pela intermitência do reforçamento negativo - FR2 ou FR3 (ver Maier e Seligman, 1976, e Peterson et al., 1993). Contudo essas propostas não se basearam em nenhuma justificativa teoricamente convincente, sendo apresentadas como "receitas" para se produzir o desamparo (ver crítica de Levis, 1976). Em oposição a elas, nossos resultados mostraram que, com uma contingência de fuga bem estabelecida, o desamparo pode ser claramente observado com ratos submetidos à situação de aprendizagem sob reforçamento negativo contínuo, o que dispensa a antiga (e infundada) restrição.

Outra discordância dos nossos resultados em comparação com os descritos na literatura diz respeito ao aspecto de "transitividade" apontado para o desamparo: há relatos de que, em ratos, o desamparo tem curta duração, desaparecendo após 48 hs da experiência com a incontrolabilidade (por exemplo, Seligman et al., 1975). Essa afirmação gerou dúvidas sobre a natureza do desamparo como fenômeno de aprendizagem (Levis, 1976). Com o nosso procedi- mento, foi obtido o desamparo até 75 dias após a exposição aos choques incontroláveis (Mestre, 1966), resultados esses que se ajustam melhor aos efeitos duradouros da aprendizagem.

Utilizando esse mesmo procedimento, obtivemos resultados divergentes sobre a importância do fator sexo dos sujeitos na aprendizagem do desamparo: ao contrário de diversos autores que afirmam que ratos fêmeas não apresentam o desamparo aprendido (Navarro et al., 1984; Steenberger, Heinsbroek, Van Haaren e Van de Poll, 1989), encontramos esse fenômeno com igual intensidade em ratos machos e fêmeas (Damiani e Hunziker, 1996). Também obtivemos analgesia condicionada induzida por choques incontroláveis sem a necessidade de re-exposição dos ratos a choques elétricos (Hunziker, 1992), ao contrário do que se afirma Jackson et al. (1979). Por fim, nos estudos que realizamos sobre os efeitos da imipramina - droga de especial interesse na área, dado seu comprovado efeito clínico como antidepressivo obtivemos repetidamente a reversão do desamparo com administrações agudas dessa droga (Graeff, Hunziker e Graeff, 1989; Gouveia Jr, 1996; Hunziker, Buonomano e Moura, 1986) ao contrário de diversos autores que defendem a necessidade de administrações crônicas para que tal reversão se dê (Sherman, Sacquitne e Petty, 1982; Petty e Sherman, 1979).

Essas diferenças sistemáticas entre os trabalhos de desamparo desenvolvidos no nosso laboratório e os publicados na literatura podem ser interpretadas de diferentes maneiras. Uma interpretação possível é que as variáveis dependentes estudadas em ambos os conjuntos de experimentos não se eqüivalem: enquanto no nosso laboratório temos evidências de que estudamos um processo de aprendizagem (de fuga), nos trabalhos que utilizam o procedimento convencional (de Maier e colaboradores) não se 
pode identificar aprendizagem associativa. Assim, é possível que nestes trabalhos, os efeitos comportamentais sejam determinados principalmente pela já comprovada depleção de alguns neurotransmissores, que leva à diminuição geral de atividade motora (Weiss et al., 1976): essas alterações neuroquímicas podem interagir com fármacos (por exemplo, a imipramina) e com os hormônios característicos de cada sexo (daí os efeitos diferenciados entre machos e fêmeas), além de retornarem à linha-de-base com o passar do tempo (e por isso a transitoriedade do efeito descrito por esses autores). É provável que as latências medidas nesses estudos, que não representam um processo de aprendizagem, sejam mais representativas da atividade motora dos sujeitos, refletindo as alterações neuroquímicas induzidas pelos choques incontroláveis. Embora esses efeitos motores provavelmente estejam presentes também nos nossos estudos, o procedimento utilizado permitiu isolar o processo de aprendizagem que, ao que tudo indica, sofre menos influência das alterações neuroquímicas apontadas por Weiss et al. (1976). Seguindo-se esta interpretação, pode-se concluir que há muito para ser feito para que a generalidade do desamparo, como fenômeno de aprendizagem, possa ser melhor avaliada.

Uma alternativa possível é analisar a relevância o controle de estímulos no desamparo aprendido. Por exemplo, Lee e Maier (1988), utilizando a aprendizagem de discriminação operante num labirinto aquático (com reforçamento negativo), obtiveram melhor discriminação por parte dos ratos expostos previamente aos choques incontroláveis que pelos animais ingênuos. Esses resultados, contrários ao previsto pela hipótese do desamparo aprendido, sugerem alterações no controle de estímulos em função da experiência com a incontrolabilidade, abrindo uma interessante alternativa de in- vestigação. Com humanos, os trabalhos sobre desamparo aprendido tem enfatizado as explicações cognitivas sobre a atribuição de causalidade feita pelo sujeito (Abranson e Seligman, 1978; Peterson et al., 1993): esses mesmos dados poderiam ser reinterpretados como comportamentos governados por regras (rulegoverned behavior), onde o desamparo seria um conjunto de comportamentos controlados pela "regra" de independência entre R e S que competiria com as contingências em vigor .

De uma maneira geral, consideramos que estudos bem conduzidos sobre o desamparo aprendido poderão trazer contribuições relevantes para a compreensão do comportamento como um todo, além da notória contribuição para o estudo do controle aversivo do comportamento e dos efeitos da não-contingência. A exploração do contínuo controlabilidade-incontrolabilidade permite a investigação de questões críticas - que incluem o confronto da contingência X contiguidade - que podem ser analisadas nos estudos do desamparo. A re-análise dos dados sobre o esse efeito, desenvolvida com rigor metodológico e conceitual, pode resgatar uma série de trabalhos bastante criativos realizados nessa área, ampliando o universo da análise do comportamento e sugerindo avanços nessas investigações.

\section{Referências Bibliográficas}

Abranson, L.Y., Seligman, M.E.P. e Teasdale, J.D. (1978).Learned Helplessness in Humans: Critique and Reformulation. Journal of Abnormal Psychology, 87, 49-74.

Alloy , 1. B. e Bersh, P.J. (1979). Partial control and learned helplessness in rats: Control over shock intensity prevents interference with subsequent escape. Animal Learning and Behavior, 7, 157-164. 
Bolles, R.C. (1970). Species-specific defense reaction and avoidance learning. Psychological Review, 77, 32-48

Damiani, K. (1993). Efeito de associações S-S sobre o desamparo aprendido em ratos machos e fêmeas: um estudo paramétrico da duração de estímulos pós-choque. Dissertação de Mestrado, Universidade de São Paulo, São Paulo.

Damiani, K. e Hunziker, M.H.L. (1996). Effects of $S$-S associations on learned helplessness: A parametric study in female and male rats. Trabalho apresentado na 22nd Annual Convention of the Association for Behavior Analysis, San Franciscค. USA.

Estes, W.K. and Skinner, B.F. (1941). Some quantitative properties of anxiety. Journal of Experimental Psychology, 29, 390-400.

Eisenstein, E.M. e Carlson, A.D. !997). A comparative approach to the behavior called "learned helplessness". Behavioural Brain Research, 86, 149-160.

Freda, J.S. e Klein, S.B. (1976). Generality of failure-to-escape helplessness phenomenon in rats. Animal Learning and Behavior, 4, 401-406.

Graeff, E.O. (1991). Efeito da administração aguda e sub-crônica da imipramina e da Bay R 1531 sobre o comportamento de fuga de ratos tratados previamente com choques incontroláveis. Dissertação de Mestrado, Universidade de São Paulo, São Paulo.

Graeff, E.O., Hunziker, M.H.L. e Graeff, F.G. (1989). Effects of ipsapirone and Bay R 1531 on learned helplessness. Brazilian Journal of Medical and Biological Research, 22, 1141-1144.

Gouveia Jr, A. (1996). Efeitos da administração aguda da imipramina sobre o desamparo aprendido em ratos machos e fêmeas. Dissertação de Mestrado, Universidade de São Paulo, São Paulo.

Hemingway, R.B.III and Reigle, T.C. (1987). The involvement of endogenous opiate system in learned helplessness and stress-induced analgesia. Psychopharmacology, 93, 353-357.

Hunziker, M.H.L (1977). Efeitos da exposição prévia a choques não-contingentes sobre a aquisição do comportamento de fuga como função de algumas dimensões da resposta. Dissertação de Mestrado, Universidade de São Paulo, São Paulo.

Hunziker, M.H.L. (1981). Um estudo sobre incontrolabilidade: considerações metodológicas, uma análise experimental. Tese de Doutorado, Universidade de São Paulo, São Paulo.

Hunziker, M.H.L. (1992). Opioid nature of learned helplessness and stress-induced analgesia observed without re-exposure to shock. Behavioural Pharmacology, 3, 117-121.

Hunziker, M.H.L., Buonomano, D.V. e Moura, M.D. (1986). Efeito da administração aguda de imipramina sobre o comportamento de ratos. Resumos de Comunicações, XVI Reunião Anual de Psicologia (pag. 35). Ribeirão Preto: SBP.

Jackson, R.L., Maier, S.F. e Coon, D.G. (1979). Long-term analgesic effects of inescapable shock and learned helplessness. Science, 206, 91-93.

Kametani, H., Nomur, S. e Shimizu, J. (1983). The reversal effect of antidepressants on the escape deficit induced by inescapable shock in rats. Psychopharmacology, 80, 206-208.

Lee, R.K.K. e Maier, S. F. (1988). Inescapable shock and attention to internal versus external cues in a water escape discrimination task. Journal of Experimental Psychology: Animal Behavior Process, 14, 302-311.

Levis, D.J. (1976). Learned helplessness: A reply and a alternative S-R interpretation. Journal of Experimental Psychology: General, 105, 47-65.

Maier, S.F., Albin, R.W. e Testa, T.J. (1973). Failure to learn to escape in rats previously exposed to inescapable shock depends on nature of escape response. Journal of Comparative and Physiological Psychology, 85, 581-592. 
Maier, S.F. e Testa, T.J. (1975). Failure to learn to escape by rats previously exposed to inescapable shock is partly produced by associative interference. Journal of Comparative and Physiological Psychology, 8, 554-564.

Maier, S.F. e Seligman, M.E.P. (1976). Learned helplessness: Theory and evidence. Journal of Experimental Psychology: General, 105, 3-46.

Martin, P., Gozlan, H. e Puech, A.J. (1992). 5-HT3 receptor antagonists reverse the helpless behavior in rats. European Journal of Pharmacology, 212, 73-78.

Mestre, M.B.A. (1996). $O$ desamparo aprendido, em ratos adultos, como função de experiências aversivas incontroláveis na infância. Dissertação de Mestrado, Universidade de São Paulo, São Paulo.

Navarro, J.I., Gil, M., Lorenzo, J.R., Noval, J., Galiana, J. e Gilbert-Rahola, J. (1984). Implicaciones de los factores no comportamentales en el modelo de desesperanza aprendida. Revista de Analisis del Comportamento, 2, 297-311.

Overmier, J.B. e Seligman, M.E.P. (1967). Effects of inescapable shock upon subsequent escape and avoidance responding. Journal of Comparative and Physiological Psychology, 63, 28-33.

Peterson C., Maier, S.F. e Seligman, M.E.P. (1993). Learned Helplessness: A Theory for the Age of Personal Control. New York: Oxford University Press.

Petty, F. e Sherman, A.D. (1979). Reversal of learned helplessness by imipramine. Communications in Psychopharmacology, 3, 371-373.

Rescorla, R.A. and Solomon, R.L. (1967). Two-process learning theory: Relationship between Pavlovian conditioning and instrumental learning. Psychological review, 74, 151-182.

Seligman, M.E.P. (1975). Helplessness: On Depression, Development and Death. Freeman: San Francisco.
Seligman, M.E.P. e Maier, S.F. (1967). Failure to escape traumatic shock. Journal of Experimental Psychology, 74, 1-9.

Seligman, M.E.P., Maier, S.F. e Solomon, R.L. (1971). Unpredictable and uncontrollable aversive events. Em E.F. Bush (Ed.). Aversive Conditioning and Learning. New York: Academic Press.

Seligman, M.E.P., Rosellini, R.A. e Kozak, M.J. (1975). Learned helplessness in the rat: time course, immunization, and reversibility. Journal of Comparative and Physiological Psychology, $88,542-547$.

Sherman, A.D., Sacquitne, J.L. e Petty, F. (1982). Specificity of learned helplessness model of depression. Pharmacology, Biochemestry and Behavior, 16, 449-454.

Skinner, B.F. (1981). Selection by consequences. Science, 213 (3), 501-504.

Steenberger, H.L., Heinsbroek, R.P., Van Haaren, F, e Van de Poll, N.E. (1989). Sex-dependent effects of inescapable shock administration on behavior and subsequent escape performance in rats. Physiology and Behavior, 45,781-787.

Weiss, J.M., Glazer, H.I. e Pohorecky, L.A. (1976). Coping behavior and neurochemical changes: An alternative explanation for the original "learned helplessness" experiments. Em G.Serban e A. Kling (Eds). Animal Models in Humam Psychology. New York: Plenum Press.

Yano, Y. (1996). Desamparo aprendido e "imunização" com diferentes respostas no treino e teste. Dissertação de Mestrado, Universidade de São Paulo, São Paulo. 